\title{
Research
}

\section{Investigating paramedic student professional identity}

Tania Johnston BScN, MHS is an Advanced Care Paramedic; and Lecturer in Paramedicine ${ }^{1 ;}$ Natalia Bilton BSc(Hon), PhD is a Lecturer in Human Anatomy and Physiology ${ }^{1}$

Affiliations:

${ }^{1}$ Charles Sturt University, School of Biomedical Sciences, Port Macquarie, New South Wales

https://doi.org/10.33151/ajp.17.759

\section{Abstract}

Introduction

A robust professional identity is a known predictor of personal and professional satisfaction and is viewed as foundational to successful practice in health occupations. Despite this, there is a gap in the research on paramedic identity (PI). Using the Macleod Clark Professional Identity Scale (MCPIS-9), the primary objective of this study was to measure the self-reported professional identity of paramedic students during their university studies. A secondary objective was to determine potential predictor variables for professional identity.

\section{Method}

A convenience sample of 102 paramedic students at a single university campus participated in this descriptive cross sectional study. An electronic, purpose designed survey recorded demographic information, background characteristics as well as the self-reported strength of student PI using the MCPIS-9.

\section{Results}

$66.7 \%$ of participants were women and $46 \%$ were between the ages of $19-24$ years; $75 \%$ reported no prior education in health care and $82 \%$ indicated paramedicine was their first choice of study. Most students $(82 \%)$ had no prior paramedic-related work or volunteer experience. The mean MCPIS-9 score was 38.8/45 with a standard deviation of 4.9. There were no statistical differences associated with PI scores and demographics or background factors, however, some interesting trends were observed in the data. The only variable found as a significant predictor of MCPIS-9 scores was the student's year of study.

\section{Conclusion}

Paramedic students in our study reported a strong sense of professional identity which had a tendency to increase from year-to-year. There appears to be a predictive relationship between year of study and strength of professional identity. We found that the selfreported strength of paramedic students' identity does not specifically correlate with gender or previous education. However, those with prior paramedic type experience who selected paramedicine as their first choice of study may experience a stronger professional identity. Further studies and subsequent replication of our findings will determine whether or not we can make solid inferences from our sample to paramedic students in general.

Keywords:

paramedic, professional, identity

Corresponding Author: Tania Johnston, tjohnston@csu.edu.au 


\section{Introduction}

An important part of being a professional is having a clear identity. A robust professional identity $(\mathrm{PI})$ is a known predictor of personal and professional satisfaction and is viewed as foundational to successful practice in health occupations (13). For example, previous research on identity demonstrates reduced attrition rates in education programs and employment for nurses with strong PI (4). Conversely, student doctors who are slow to develop a strong identity can struggle to transition to the medical profession (5). High measures of $\mathrm{PI}$ are also a partial determinant of effective interprofessional learning and collaboration in health professions $(6,7)$.

Professional identity encompasses the shared knowledge, skills, values, beliefs and attitudes specific to a professional group $(4,8,9)$. Despite its importance, very little is known about $\mathrm{PI}$ in the paramedic profession. In the small amount of paramedic research available, Donnelly et al propose a role identity scale to measure paramedic stress and burnout (10). Other studies explore paramedics' PI during the transition from clinical practice to academia (11) and students' professional socialisation into the workplace (12). This paucity of evidence represents a significant gap at a time when the discipline is in the midst of becoming a formally recognised health profession in Australia.

\section{By joining the Australian Health Practitioner Regulatory}

Agency in 2018, Australian paramedics have now attained national registration alongside other established professions in health $(13,14)$. At the same time, the scope of practice and role expectations of a paramedic practitioner are evolving as paramedicine responds to changes and demands in health care $(15,16)$. This contributes to an ongoing tension for paramedics who have traditionally struggled to define their identity (1721). Developing and maintaining a strong $\mathrm{Pl}$ is required for practitioners to fully accept and perform the responsibilities of a professional role (5). This is imperative in the context of role changes where identity confusion may ensue and potentially erode paramedic confidence, leading to compromise work practices $(22,23)$.

Though it is generally accepted that identity and work role changes are interrelated, exactly how and when professional identity is formed is poorly understood (24). Students entering a program of study will start with some measure of $\mathrm{PI}$ and there is agreement that universities play an important role in fostering this identity to strengthen students' career preparation $(2,4,25-28)$. As paramedic students' progress through their didactic and practical education, they will internalise the values and attributes of the profession (5). Their professional identity is transformational and subsequently influenced by educators and mentors, workplace learning experience and professional socialisation $(2,25,26)$. This study serves to contribute to a better understanding of paramedic student PI in the current context of professionalisation and ongoing changes to the practitioner role $(15,19,29)$.

\section{Theoretical framework}

As a social construct, identity is a nebulous concept. In his work on organisations, Weick explains identity as a lens we look through to help make sense of the world around us (30). Theories on identity help us understand how people socially interact in society and how to interpret the relationship between individual behaviours and social structure (31). Foundational to PI, people have a tendency to ascribe to the particular characteristics of a group to which they feel an emotional attachment and sense of belonging (32).

Professional identity can be examined through the Social Identity Theory (SIT) framework. SIT focusses on group processes and intergroup relations, bridging the gap between an individual's self-concept and the social groups they belong to (33). Whereas Identity Theory is primarily aimed at self and how individuals occupy roles in society, SIT is helpful to better understand identity that forms with group membership such as within a profession (34). SIT has been used in health research to better understand how and why people categorise themselves in certain ways including their chosen profession $(27,33)$. This makes it a useful framework in our study which is focussed on measuring how students rate their alignment to paramedics as a professional group.

In summary, there is an identified gap in the literature regarding paramedic professional identity; a topic that is especially important in the context of professionalisation. Given the importance of university education in shaping one's professional identity, it is valuable for academics to understand how students report the strength of their PI. Using the Macleod Clark Professional Identity Scale (MCPIS-9) tool, the primary objective of this study was to measure the selfreported professional identity of paramedic students during their university studies. A secondary objective was to determine potential predictor variables for student professional identity.

\section{Methods}

\section{Participants}

This descriptive study used a cross-sectional survey approach to gather data. Cross-sectional surveys are able to provide snapshots of populations at a specific point in time (35). Our research setting was a single campus of Charles Sturt University, an Australian regional university. We recruited participants as a convenience sample of students attending the first lecture of the session at the start of the year in three respective subjects for each cohort. Our primary purpose in using this sampling strategy was to recruit first year students just as they started the session and were not yet influenced by the paramedic curriculum, lecturers or other program activities. Students were given a participant information sheet and invited 
to participate via a link to SurveyMonkey (SurveyMonkey, Palo Alto, CA, USA). We did not compensate students in any way, and they were assured in the participant information sheet that responses were deidentified and that a decision to participate or not would have no impact on their academic standing.

\section{Procedure}

Participants in this study were invited to participate in the study during class time in 2016 across a single academic session of study. The rationale behind recruiting participants during class time was to avoid the low response rate commonly associated with email invitations, to ensure that the demographic profile of the survey reflected that of the target population, and to provide a sufficiently large data set for meaningful statistical analysis (36). Those students that chose not to participate in the study were given the option to undertake personal quiet reading time if they so wished.

Two paramedic students were included on the research team per the study protocol and did not participate in the survey. Along with the primary investigator, they provided the students a brief introduction about the purpose of the research. All members of the research team exited the lecture theatre to reduce any influence on participation rates. This was the first time our students had been asked to participate in research on professional identity. A non-academic staff member explained to the students how to access SurveyMonkey® via a link projected onto the lecture screen. Students used their own electronic devices to access the link which opened the participant information sheet. Consent was assumed if students progressed into the study.

The purpose designed survey collected demographic information and included 12 multiple choice, binary yes or no, and Likert scale questions. The instrument was devised to assess how strongly the students identified with the paramedic profession and what influences they may have had before studying paramedicine. Participants were asked whether paramedicine was their first choice of study, if they had other post-secondary education, and whether they have family or close friends who are paramedics, doctors or nurses. The survey concluded with the MCPIS-9 to assess the students' identification with studying and being a member of the paramedic profession. Participants completed the entire survey in less than 10 minutes.

\section{Measurement}

In a research field dominated by nursing, the MCPIS- 9 is one of two known measures of PI specifically designed for allied health (37). Crossley and Vivekananda-Schmidt also developed a PI measure for non-nurses, the Professional Self Identity Questionnaire (PSIQ). The PSIQ was created to measure PI in health, social work and medical students (37) but unlike the MCPIS-9, the tool has not been validated in further studies.
Historically, Brown et al first used a 12-item scale to measure occupational identity among factory workers (38). Building on this work, Adams et al subsequently developed their nine item MCPIS-9 scale to measure the professional identity of first year health and social work students $(n=1254)$ who were undertaking interprofessional education (27). A quantitative survey instrument, the MCPIS-9 includes statements asking respondents to rate how they feel about belonging to and identifying with a professional group.

After piloting the tool and validating it through an expert panel, Adams et al performed an exploratory factor analysis (EFA) resulting in a one-component nine-item solution. They reported the MCPIS- 9 to have an adequate internal reliability of 0.79 (Cronbach's alpha) where greater than 0.7 is considered an acceptable measure of internal consistency $(35,39)$. In a later study, Worthington et al evaluated the predictive validity of this scale for students transitioning to the nursing profession. Similarly, their EFA determined a one-component solution while the Cronbach's alpha was reported as 0.83 (4).

In contrast, Cowen et al identified problems with the MCPIS-9 scale. In their research involving nursing students, they reported poor results from a confirmatory factor analysis and found the tool to be a three-factor model of identity rather than one as previously described. The Cronbach's alpha confirmed internal consistency at 0.78 (3). In their evaluation of five PI tools, Cowen et al concluded that more research is needed to develop a psychometrically strong measure of professional identity.

To date, there are no specific professional identity measures available that have been used in paramedicine. In broader terms, the evidence on psychometric evaluation is reported to be generally lacking and there are concerns regarding reliability, internal consistency and structural validity of the current measurement tools (37). Despite reported weaknesses, we selected the MCPIS-9 professional identity measure from the limited number of instruments available to health researchers outside of nursing that have been used in more than one study.

The MCPIS-9 required students to answer questions using a 5 -point Likert scale (strongly disagree $=1$, to strongly agree $=$ 5). The scale produces scores ranging from a minimum of nine through to a maximum of 45 . The higher the score, the higher the participant's self-reported level of professional identity. The MCPIS-9 includes statements phrased in the positive and negative to help correct errors in random measurement (27).

\section{Ethics approval}

The study was approved by the Charles Sturt University Human Research Ethics Committee (approval number 400/2016/01). 


\section{Data analysis}

There were two goals to the data analyses. Part one was focussed on describing the cohort data overall and as a function of year of study. Part two involved analysing the MCPIS-9 scores as they relate to the factors outlined in Figure 1.

The data was tabulated, and descriptive statistics calculated using the frequencies dialog in IBM SPSS Statistics for Windows, Version 25.0. The researchers coded the MCPIS-9 Likert scale data to properly reflect scoring for negatively phrased questions.

\section{Results}

\section{Part 1. Cohort demographics}

The response rate for this survey was $89.5 \%$ overall. By cohort, the response rates were $92.7 \%$ for first years, $100 \%$ for second years and $70.4 \%$ for third year students. The majority of respondents were female between the ages of 19-24 years. The majority reported no prior education in health care and agreed that paramedicine was their first choice of study. Most students had no prior paramedic- related work or volunteer experience. The characteristics of the study sample of this data are shown in Table 1.

Participants were asked to indicate if they had paramedics, nurses or doctors as family members or friends. Most of the respondents have a family member in the health profession, primarily in nursing. Furthermore, most respondents stated that they have a friend in the healthcare field, and again this was primarily in nursing (Table 2).

\section{Part 2. MCPIS-9 scores analysis}

Of the 102 participants across the three cohorts, 96 completed the MCPIS-9. The range of MCPIS-9 scores was 15 to 45 with a mean score of 38.77 and a standard deviation of 4.89 (Figure 1). The Cronbach's alpha for the MCPIS- 9 scale in our study was 0.84 . Boxplots of the data revealed two outliers in the first-year data (scores of 15 and 18) which were removed before subsequent statistical analyses. We conducted preliminary tests of our data to evaluate homogeneity of variance and normality of scores. As our data violated the assumptions of normality with regards to the distributions of scores and the assumption of homogeneity of variance between groups, we performed non-parametric tests as appropriate.

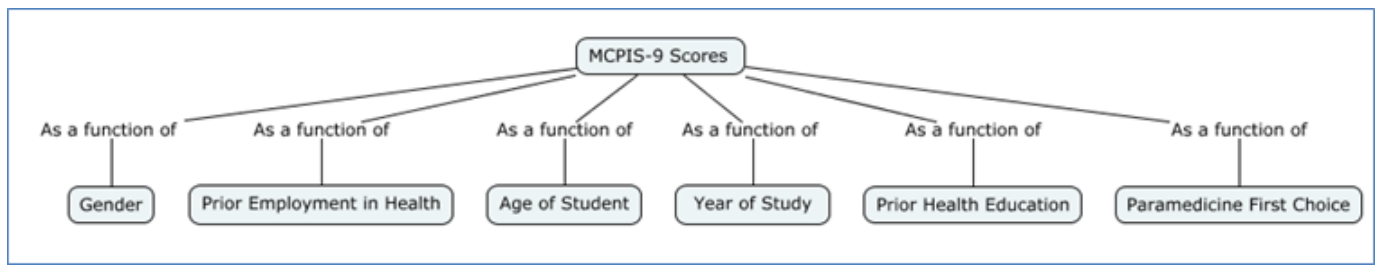

Figure 1. Factors used to statistically test the MCPIS-9 scores

Table 1. Demographic information for the entire cohort $(n=102)$ and divided by the students' year of study. The data are the mode in each case with (percentage of responses)

\begin{tabular}{|c|l|c|c|c|c|}
\hline Q\# & \multicolumn{1}{|c|}{ Question } & $\begin{array}{c}\text { All years } \\
(\mathbf{n = 1 0 2})\end{array}$ & $\begin{array}{c}\text { First year } \\
(\mathbf{n}=51)\end{array}$ & $\begin{array}{c}\text { Second year } \\
(\mathbf{n = 3 2})\end{array}$ & $\begin{array}{c}\text { Third year } \\
(\mathbf{n}=19)\end{array}$ \\
\hline 1 & What is your gender? & Female $(66.7 \%)$ & Female $(64.7 \%)$ & Female $(68.8 \%)$ & Female $(68.4 \%)$ \\
\hline 2 & What age group are you in (in years)? & $19-24(46.1 \%)$ & $19-24(35.3 \%)$ & $19-24(56.3 \%)$ & $19-24(58.9 \%)$ \\
\hline 4 & Do you have any prior education in health? & No $(74.5 \%)$ & No $(70.1 \%)$ & No $(75.0 \%)$ & No $(84.2 \%)$ \\
\hline 7 & Was paramedicine your first choice of study? & Yes $(82.0 \%)$ & Yes $(81.6 \%)$ & Yes $(84.4 \%)$ & Yes $(78.9 \%)$ \\
\hline 8 & $\begin{array}{l}\text { Did you have prior employment or volunteering } \\
\text { experience in paramedicine-type practice? }\end{array}$ & No $(82.0 \%)$ & No $(87.9 \%)$ & No $(81.3 \%)$ & No (68.4\%) \\
\hline
\end{tabular}

Table 2. Responses to questions asking about friends and family members, all years combined and divided by answer options (paramedics, nurses or doctors). Respondents selected all that applied.

\begin{tabular}{|c|l|c|c|c|c|}
\hline Q\# & \multicolumn{1}{|c|}{ All years combined } & Total & Paramedics & Nurses & Doctors \\
\hline 10 & $\begin{array}{l}\text { Indicate if any of your family members are or were employed as } \\
\text { paramedics, nurses, doctors }\end{array}$ & 42 & 2 & 36 & 8 \\
\hline 11 & $\begin{array}{l}\text { Indicate if any of your friends are or were employed as paramedics, } \\
\text { nurses, doctors }\end{array}$ & 61 & 26 & 54 & 15 \\
\hline
\end{tabular}


Figure 2 shows the combined MCPIS-9 scores and by individual cohort. For participants in their first year of study $(n=51)$, the mean MCPIS-9 score was $37.8(S D=5.8)$. For participants in their second year of study $(n=32)$, the mean MCPIS-9 score was 39.1 (SD=3.4), and for participants in their third year of study $(n=19)$, the mean MCPIS-9 score was 40.7 $(S D=3.8)$. A Kruskal-Wallis ANOVA indicated that the effect of year of study on MCPIS-9 score neared but did not reach statistical significance in our study $(H=5.449, d f=2, N=96$, $\mathrm{p}=.066)$.

A Kruskal-Wallis ANOVA also indicated that there were no statistically significant differences between the age of student and MCPIS-9 score $(H=3.649, d f=5, N=91, p=.601)$. For the age groups 18 years or less: $19-24,25-29,30-34,35-39$ and $40-44$, the mean ranks were $45.4,47.7,41.3,58.1,35.4$ and 59.3 , respectively.

Next, Mann Whitney U tests revealed that the MCPIS-9 scores for males (mean 49.45) was not statistically different to that of females (mean 44.31, $U=812, z=-.876, p=.381$ ). Though students with prior employment or volunteer experience in health-related roles did report higher MCPIS-9 scores than those that did not state that they had this experience (52.0 vs. 44.6) this failed to reach statistical significance $(U=527$, $z=-1.046, p=.296)$. The students that stated that they did have prior education in a health-related field scored similarly and therefore were not statistically different from those that indicated that they did not have any prior education in health (45.6 vs. $45.2, \mathrm{U}=726, \mathrm{z}=-.090, p=.928$ ). Students whose first choice of study was paramedicine scored higher than those students that stated otherwise in the survey. Again, this difference (47.2 vs. 39.2) did not reach statistical significance $(U=418, z=-1.016, p=.309)$.

As a final test we employed a stepwise linear regression to determine if MCPIS-9 scores could be predicted from the variables included in this study. The analysis showed that there was only one predictor variable that was significant in our model. This predictor was year of study with a coefficient of 0.857 . The resultant equation was MCPIS-9 score $=38.1+$ 0.857 (year of study), $(F(1,89)=4.059, p=.047)$.

\section{Discussion}

The objectives of this study were to measure the PI of university student paramedics and to determine potential predictor variables for student PI. It also presented an opportunity to report on the demographics and background of these participants who represent a cohort of paramedic students at a single university campus. Overall, our students reported high levels of $\mathrm{PI}$ across all years and our study results gave us valuable insight we can use to strengthen aspects of PI within our paramedic curriculum.

Tan, Van der Molen and Schmidt propose that university educators who aim to develop PI should focus on five distinct dimensions. The first three include providing practice knowledge, professional experience, and role modelling to help students understand what it means to be in the profession and what the expectations are. Next, professional self-efficacy and

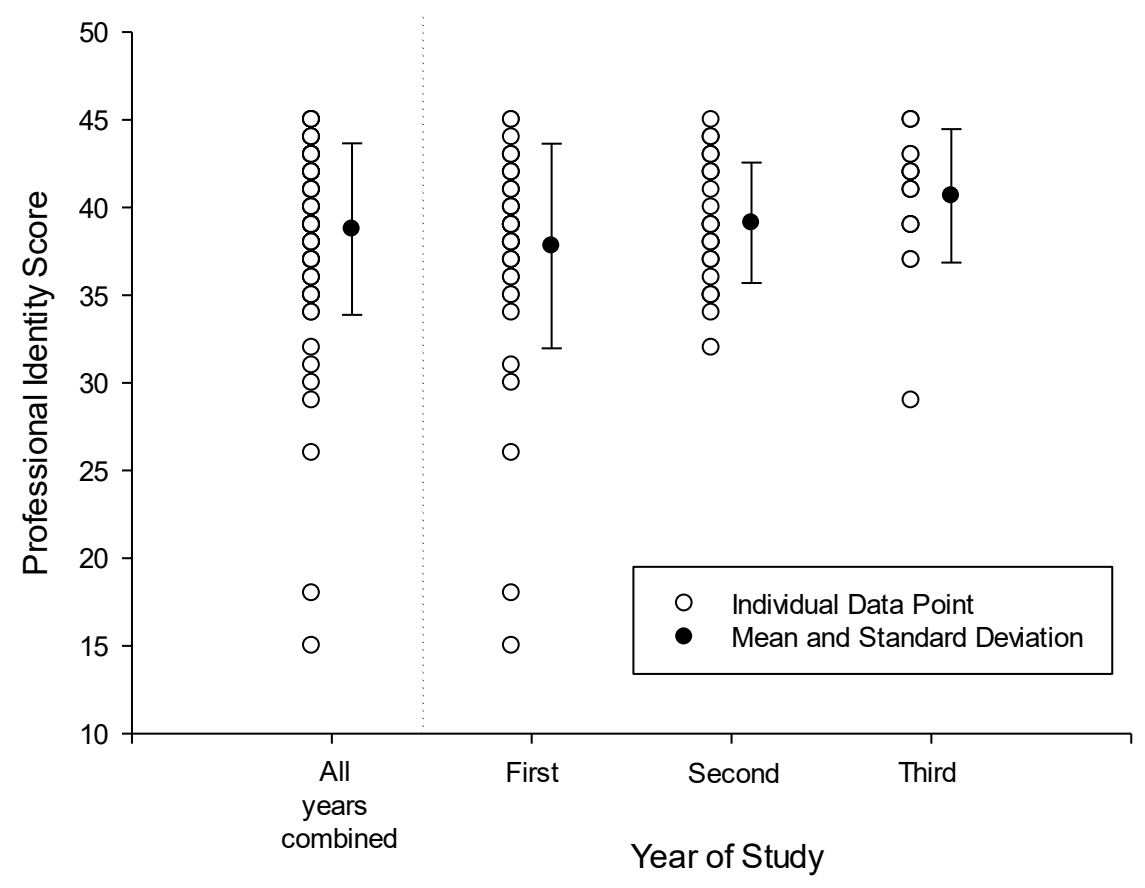

Figure 2. Professional identity scores (MCPIS-9) for first, second and third year students combined and divided by year of study 
preference for the profession are two dimensions that signal student readiness and confidence as well as how strongly they identify with learning about profession (28). Whereas equipping students with professional knowledge and workplace experience is core business for universities, influencing the remaining dimensions presents a more complex challenge. Our results validated our students' strong preference for the paramedic profession and increased our awareness of the students' lack of prior exposure to paramedic role models.

The results of our study revealed that our paramedic students across all years identified strongly with their profession during their first lecture of the session. Using the MCPIS-9, the participants reported an overall average score of 38.8 out of a possible $45(\mathrm{SD}=4.9)$. Additionally, we determined that this high affinity does not diminish year-on-year, an encouraging finding that we can explore with future qualitative research. Our results also demonstrated that the vast majority of our students $(82 \%)$ selected paramedicine as their first choice of study, signalling that they entered university with a strong preference for the profession.

Next, our study highlighted the paucity of paramedic role modelling available to our students before entering university. Of interest is that most (82\%) of our students stated that they chose paramedicine despite having no prior exposure to paramedic-related volunteerism or work. Relatively few identify having paramedic family members $(2 \%)$ or friends $(25 \%)$. Most of our participants appear to have had little engagement with members of the paramedic profession before their studies, though over half have friends in nursing. Though more research is needed, these results bring in to question whether our students have been influenced more by the nursing profession rather than paramedicine. This emphasises how important it is for universities to provide students with opportunities to observe and interact with paramedic role models. Role models may include clinically experienced lecturers as well as clinicians who are currently practising (28). Acknowledging the role of hidden curricula, students are also inevitably influenced by peer tutors and fellow students while any interactions with lecturers, clinicians and students can have either a positive or negative impact on their $\mathrm{PI}(40)$. It follows that our students with generally high MCPIS-9 scores should be receptive to formal role modelling in our curricula, further strengthening their PI.

When we analysed our cohort data for predictor variables we did not find a statistical difference in reported PI scores between genders, between those with previous health education, paramedic-type work or volunteer experience, nor between those who stated that paramedicine was their first choice of study. Though not statistically significant, we did note an upward trend in MCPIS-9 scores for those who did report paramedicine as their first choice and for those students with prior paramedic related experience. This can be compared to Worthington et al who revealed that female nurses had a stronger PI, especially if they had prior education in health or work experience in the field of nursing (4). Similarly, Crossley and Vivekananada-Schmidt reported that previous experience in health had a positive impact on medical students' PI (5). Students across 10 health professions in the study by Adams et al also reported stronger $\mathrm{PI}$ if they had prior employment or volunteer experience in a related field (27). Though more research is needed, our findings suggest that paramedic students are similar to nursing and other health professions in that they are more likely to have a stronger professional identity if they have been exposed to the occupation and then specifically pursue it as their chosen career. This information could serve to strengthen our recruitment strategies by encouraging potential applicants to seek industry-related experience before applying.

Our results did demonstrate a slight trend towards increased professional identity as students' progress in their studies (first year $M=37.8(n=51)$, second year $M=39.1(n=32)$, third year $M=40.7(n=19))$. This did not prove to be statistically significant, however, the step wise analysis did show that a student's year of study was a significant predictor of professional identity. Our finding suggests that students with a lower sense of professional identity may be more likely to leave university in first or second year as compared to those who progress to completion. Further research would be needed to validate this assertion and to better understand paramedic student attrition .

Finally, our study also presented an opportunity to report on the demographics and background of these participants who represent a cohort of paramedic students at a single campus. For comparison, we found that the majority of our paramedic student cohort is female $(66.7 \%)$ while others who have researched professional identity in health reported an even higher percentage of female students $(82-84 \%)(4,27,41)$ common age range $(46 \%)$ compared to the aforementioned studies that reported an average age of 25.2 years (27), 21.5 years (41) and 25.2 years (4). The vast majority of this cohort of paramedic students have no prior education in health (74.5\%) which is consistent with Adams et al and Worthington et al whose participants also reported no to this question $(84.1 \%$ and $78 \%$ respectively) $(4,27)$. When viewed as a whole, these demographic findings demonstrate that our paramedic student cohort is substantially similar to other health students who were participants in similar studies on PI. This is useful given the paucity of paramedic literature and the reliance we have on applying research from nursing and other health professions.

\section{Our study on paramedic student professional identity} represents research in an area that has been identified as a weakness in paramedicine and provides a benchmark for future studies. This is a valuable contribution as research in paramedicine gains momentum alongside other more established health professions. Research on paramedic identity is limited and to date, none have been done on students using a measurement tool such as the MCPIS-9. Our study explored 
potential factors that can potentially influence professional identity in paramedic students and serves to inform paramedic educators who play an important role in fostering student identity.

\section{Limitations}

This study has several limitations. Our sample only represents paramedic students enrolled in one regional university at a single point in time and may not be generalisable due to variation in student cohorts and educational programs. Future studies involving multiple universities nationally or internationally would increase the ability to generalise findings. Our findings on PI should be interpreted with caution as the study instrument relies on self-report data and whose psychometric properties require further validation. Next, the recruitment strategy was likely responsible for the high response rate but potentially limited our access to participants in the third-year cohorts who did not attend the first lecture of the session. In addition, students who attended the first lecture and answered the survey may be more inclined to reporting higher PI than those who did not attend.

Future research using a longitudinal approach would be useful to better understand how identity evolves as a result of paramedic role modelling, the hidden curricula and other university influences. Finally, additional studies could aim to demonstrate whether professional identity scores correlate with attrition rates or even successful transition into the paramedic workforce.

\section{Conclusion}

Our findings indicate that paramedic students in our study report a strong sense of professional identity when they enter university and that this tended to increase over the course of their studies. This study is the first of its kind to show a predictive relationship between year of university study and strength of paramedic student PI. We found that students with exposure to paramedic-related experience who selected paramedicine as their first choice of study may experience a stronger professional identity. We also determined that our students are not likely to have paramedic role models available to them before university, emphasising the importance of role modelling in our curricula. Finally, our study revealed similar characteristics in terms of gender, age and previous education in health between our cohort of paramedic university students as compared to similar studies involving nursing and allied health students. This is something that is useful when assessing the generalisability of available research findings on identity. Given the important role that universities play in fostering professional identity, these results serve to inform educators responsible for developing paramedic curriculum and workplace learning experiences.

\section{Competing interests}

The authors declare no competing interests. Each author of this paper has completed the ICMJE conflict of interest statement.

\section{Acknowledgements}

The authors would like to thank paramedic students S. Graham and $\mathrm{H}$. Stack for their contributions during data collection.

\section{References}

1. Monrouxe LV. Negotiating professional identities: dominant and contesting narratives in medical students' longitudinal audio diaries. Current Narratives 2009;1:41-59.

2. Cook TH, Gilmer MJ, Bess CJ. Beginning students' definitions of nursing: An inductive framework of professional identity. J Nurs Educ 2003;42:311.

3. Cowin LS, Johnson M, Wilson I, Borgese K. The psychometric properties of five professional identity measures in a sample of nursing students. Nurse Educ Today 2013;33:608-13.

4. Worthington M, Salamonson Y, Weaver R, Cleary M. Predictive validity of the Macleod Clark Professional Identity Scale for undergraduate nursing students. ibid. 2013;33:187.

5. Crossley J, Vivekananda-Schmidt P. The development and evaluation of a Professional Self Identity Questionnaire to measure evolving professional self-identity in health and social care students. Med Teach 2009;31:e603-7.

6. Parsell G, Bligh J. The development of a questionnaire to assess the readiness of health care students for interprofessional learning (RIPLS). Med Educ 1999;33:95100.

7. Hind M, Norman I, Cooper S, et al. Interprofessional perceptions of health care students. J Interprofess Care 2003;17:21-34.

8. Hercelinskyj G, Cruickshank M, Brown P, Phillips B. Perceptions from the front line: professional identity in mental health nursing. Int J Ment Health Nurs 2014;23:2432.

9. Goltz HH, Smith ML. Forming and developing your professional identity. Health Promot Pract 2014;15:785-9.

10.Donnelly EA, Siebert D, Siebert C. Development of the Emergency Medical Services Role Identity Scale (EMSRIS). Soc Work Health Care 2015;54:212-33.

11. Munro GG, O'Meara P, Mathisen B. Paramedic academics in Australia and New Zealand: the 'no man's land' of professional identity. Nurse Educ Pract 2018;33:33-6.

12.Devenish S, Clark M, Fleming M, Loftus S. The professional socialisation of paramedics: The transition from university student to practising paramedic. Int J Qual Methods 2012;11(5):784-5. 


\section{References (continued)}

13. Williams B, Brown T, Onsman A. From stretcher-bearer to paramedic: the Australian paramedics' move towards professionalisation. J Emerg Prim Health Care 2009;7:8.

14. Gough S. Paramedics: Welcome to the National Scheme! 2018. Available at: www.ahpra.gov.au/News/2018-11-30Paramedics-Welcome-to-the-National-Scheme.aspx

15. Joyce CM, Wainer J, Piterman L, Wyatt A, Archer F. Trends in the paramedic workforce: a profession in transition. Aust Health Rev 2009:33:533-40.

16.O'Brien K, Moore A, Dawson DA, Hartley PR. An Australian story: paramedic education and practice in transition. Australasian Journal of Paramedicine 2014;11:4.

17. Gilmour B. The EMS identity crisis. EMS World [Internet]. 2014. Available at: www.emsworld.com/article/11672173/ guest-editorial-the-ems-identity-crisis

18. Hsieh A. Staffing problems will be solved when EMS finds its identity. 2014. Available at: www.ems1.com/emsmanagement/articles/1929981-Staffing-problems-will-besolved-when-EMS-finds-its-identityl

19. O'Meara P. So how can we frame our identity? J Paramedic Practice 2011;3:57.

20.McCann L, Granter E, Hyde P, Hassard J. Still bluecollar after all these years? An ethnography of the professionalization of emergency ambulance work. Journal of Management Studies 2013;50:750-76.

21.Johnston T, Acker J. Using a sociological approach to answering questions about paramedic professionalism and identity. Australian Journal of Paramedicine 2016;13(1).

22. Machin Al, Machin T, Pearson P. Maintaining equilibrium in professional role identity: a grounded theory study of health visitors' perceptions of their changing professional practice context. Journal of Advanced Nursing 2012;68(7):1526-37.

23.McDonald R. Individual identity and organisational control: empowerment and modernisation in a primary care trust. Sociol Health IIIn 2004;26:925-50.

24.Pratt M, Rockmann K, Kaufmann J. Constructing professional identity: the role of work and identity learning cycles in the customization of identity among medical residents. Acad Manage J 2006;49:235-62.

25.Mylrea MF, Sen Gupta T, Glass BD. Developing professional identity in undergraduate pharmacy students: a role for selfdetermination theory. Pharmacy (Basel) 2017:5:16.

26.Jackson D. Re-conceptualising graduate employability: the importance of pre-professional identity. Higher Education Research \& Development 2016;35:925-39.

27.Adams K, Hean S, Sturgis P, Clark JM. Investigating the factors influencing professional identity of first-year health and social care students. Learning in Health and Social Care 2006;5:55-68.

28. Tan CP, Van Der Molen HT, Schmidt HG. A measure of professional identity development for professional education. Studies in Higher Education 2017;42:1504-19.

29. O'Meara P, Tourle V, Stirling C, Walker J, Pedler D. Extending the paramedic role in rural Australia: a story of flexibility and innovation. Rural Remote Health 2012;12:1978.

30.Weick KE. Sensemaking in organizations. Thousand Oaks, CA: Sage; 1995.

31. Hogg MA, Terry DJ, White KM. A tale of two theories: a critical comparison of identity theory with social identity theory. Soc Psychol Q 1995;58:255-69.

32.Van Knippenberg D. Work motivation and performance: a social identity perspective. Applied Psychology 2000;49:357-71.

33.Burford B. Group processes in medical education: learning from social identity theory. Med Educ 2012;46:143-52.

34.Stets J, Serpe R. Identity Theory. Handbook of Social Psychology. Dordrecht, NL: Springer Science and Business Media; 2013. p. 31-60.

35. Encyclopedia of Survey Research Methods. Thousand Oaks, CA: Sage Publications; 2008.

36. Kelley K, Clark B, Brown V, Sitzia J. Good practice in the conduct and reporting of survey research. Int J Qual Health Care 2003;15:261-6.

37. Matthews J, Bialocerkowski A, Molineux M. Professional identity measures for student health professionals - a systematic review of psychometric properties. (Report). BMC Med Educ 2019;19(1).

38. Brown R, Condor S, Mathews A, Wade G, Williams $J$. Explaining intergroup differentiation in an industrial organization. Journal of Occupational Psychology 1986;59:273-86.

39.Nunnally JC. Psychometric theory. N.Y: Mcgraw-Hill; 1967.

40.McKenna L, Williams B. The hidden curriculum in near-peer learning: an exploratory qualitative study. Nurse Educ Today 2017; 50:77-81.

41. Carpenter J. Doctors and nurses: Sterotypes and stereotype change in interprofessional education. J Interprof Care 1995;9:151-61. doi:10.3109/13561829509047849 